\title{
STRUCTURAL TRANSITIONS IN BIOPOLYMERS: FROM DNA TO PROTEIN TO SPIDER SILK
}

\author{
HAIJUN ZHOU* \\ Institute of Theoretical Physics, the Chinese Academy of Sciences, \\ Beijing 100080, China \\ *E-mail: zhouhj@itp.ac.cn
}

\begin{abstract}
Biopolymers are ubiquitous in a biological living system. They are involved in almost all cellular biochemical reactions, playing versatile biological roles. The mechanical characteristic of a biopolymer is directly related to its functions. In this paper, we briefly review theoretical efforts of our group on the structural transitions of three major types of biopolymers (DNA, RNA, proteins) and on the structural organization in the spider capture silk. These theoretical studies demonstrated that, weak non-covalent interactions, such as wan der Waals attraction, hydrogen bonding, hydrophobic interaction, and screened electrostatic interactions, enable a biopolymer to achieve an optimal combination of structural stability and deformability in accordance with its specific biological functions.
\end{abstract}

\section{Introduction}

At physiological conditions, the typical force involved in a biological process is of the order of piconewtons $\left(1 \mathrm{pN}=10^{-12} \mathrm{~N}\right)$. Such high levels of force resolution have been achieved in the last two decades by using singe-molecule manipulation methods. For example, a magnetic tweezer can generate a constant force in the range $0.01 \mathrm{pN}-100 \mathrm{pN}$, an optical tweezer can generate forces $0.1 \mathrm{pN}-150 \mathrm{pN}$, and an atomic force microscopy can generate forces $5 \mathrm{pN}-10 \mathrm{nN}\left(1 \mathrm{nN}=10^{-9}\right.$ $\mathrm{N}=10^{3} \mathrm{pN}$ ). With these new instruments, the response of a single biopolymer under the action of an external force can be measured and structural transitions in a biopolymer can therefore be detected. The first biopolymer to be studied this way is the double-stranded DNA (dsDNA), the genetic material of a biological organism $^{1}$. Later on, the mechanical properties of single-stranded DNA (ssDNA), RNA, and protein molecules such as titin and silk proteins were also studied. These were followed by single-molecular studies on the interactions between DNA and various proteins, including DNA polymerases, DNA topoisomerases, helicases, and other motors/enzymes. The structural organizations of eukaryotic chromosomes were also probed by mechanical experiments.

Biopolymers DNA, RNAs, and proteins are the most important macromolecules in a living cell. DNA stores the genetic information of a cell. This information is translated into protein sequences by way of RNAs. After a protein sequence is folded into its native three-dimensional structure, it is then ready to perform 
its biological roles (such as gene-expression regulation, signal transduction, etc.). There are many very complicated physical interactions among DNA, RNAs, and proteins. The mechanical property of a biopolymer is therefore directly related to its functions. The accumulation of single-molecular force manipulation data make it possible to gain quantitative understanding of the mechanical properties of these important biopolymers. In the last several years, we have performed a series of theoretical investigations on the structural transitions of DNA, RNAs, and proteins. We have also studied the elasticity of spider capture silk. These theoretical studies demonstrated that, weak non-covalent interactions, such as wan der Waals attraction, hydrogen bonding, hydrophobic interaction, and screened electrostatic interactions, enable a biopolymer to achieve an optimal combination of structural stability and deformability in accordance with its specific biological functions.

This paper is a brief review of our own theoretical efforts. The review article Ref. 2 contains an expanded overview of the theoretical progresses on singlebiopolymer modeling. For readers who wishes to get an overview of the experimental development, the review articles Refs. 3, 4, 5, 6, 7 can be consulted.

This review paper is organized as follows. We first mention three simple models for biopolymers in the next section. In Sec. 3 the mechanical property of dsDNA is studied; followed by the RNA and ssDNA secondary structure denaturation in Sec. 4. Section 5 investigate the unfolding process of protein $\beta$-sheet secondary structures. In Sec. 6 we present a hierarchical chain model to account for the exponential force-extension relationship of spider capture silk. The last section concludes this paper.

\section{Simple polymer elasticity models}

As a starting point, we list in this section the major properties of three simple polymer models. Related information on polymer elasticity models can be found in text books such as Ref. 8 and Ref. 9. Due to sequence heterogeneity and complicated intra-polymer and inter-polymer interactions, real biopolymers are of cause more complex than these simple models. By comparing the behavior of a real biopolymer with those of the model polymers, we may be able to distinguish mechanical properties that are specific to this biopolymer from mechanical properties that are general to all polymers.

\subsection{The Gaussian chain model}

The simplest polymer model is the Gaussian chain model. This model regards a polymer as composed of $N$ beads. At time point $t$, each bead $i$ is in position $\mathbf{r}_{\mathbf{i}}(\mathbf{t})$; between two consecutive beads $i-1$ and $i$ there is the following harmonic interaction

$$
E_{i-1, i}=\frac{3}{2} \frac{k_{B} T}{b^{2}}\left(\mathbf{r}_{i}-\mathbf{r}_{i-1}\right)^{2},
$$

where $k_{B}$ is Boltzmann's constant, $T$ is the temperature, and $b$ is the Kuhn length. The Kuhn length $b$ characterizes the mean square root distance between bead $i-1$ 

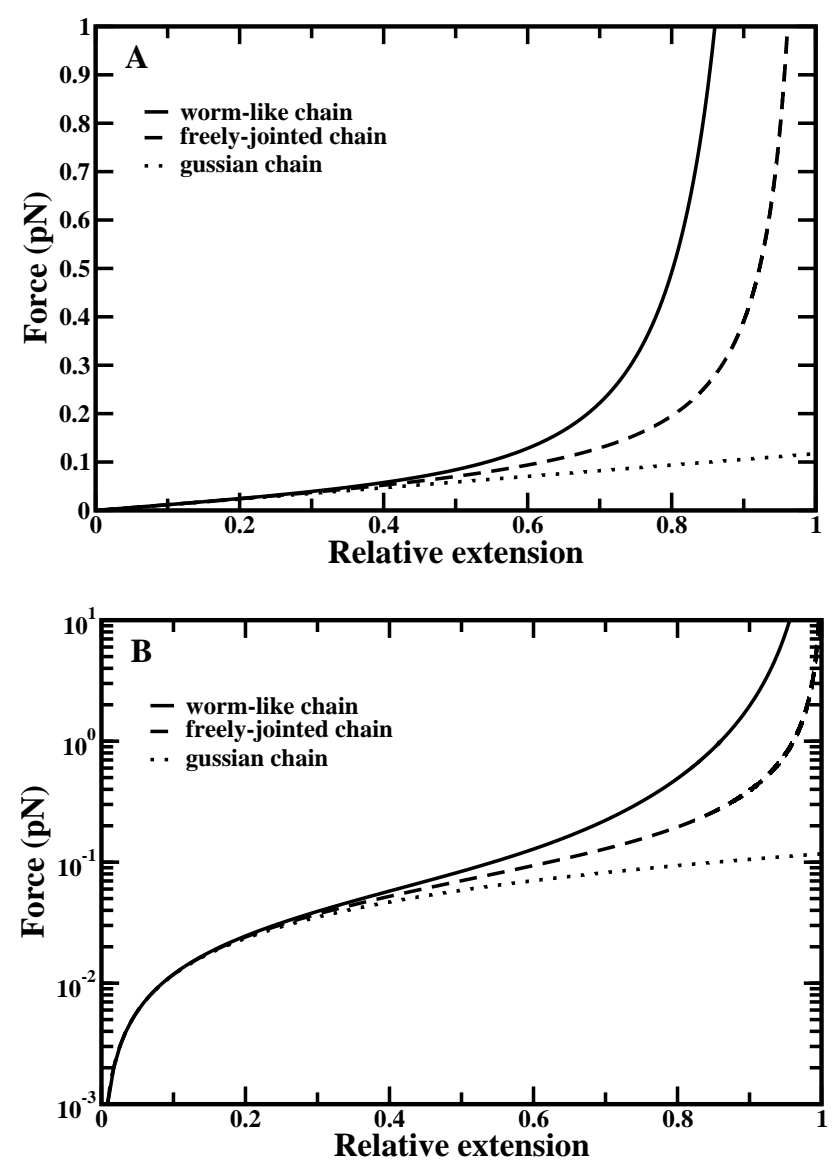

Figure 1. Force-extension relations for a Gaussian chain (dotted line), a freely-jointed chain (dashed line), and a wormlike-chain (solid line). All three curves are produced with the same Kuhn length $b=100 \mathrm{~nm}$ and the same temperature $T=300 \mathrm{~K}$. In (A), force is in linear scale while in (B), force is in logarithmic scale.

and bead $i$ in the absence of external force. Under the action of an external force of magnitude $f$, the extension $z$ of the Gaussian chain along the force direction is

$$
z=\frac{N b}{3} \frac{b f}{k_{B} T} .
$$

This is a linear force-extension relation (see the dotted lines of Fig. 1). From Eq. (2), one know that the characteristic length scale of the Gaussian chain is $b$, and the characteristic force scale of the Gaussian chain is $k_{B} T / b$. For a biopolymer such as RNA and proteins, the Kuhn length $b$ is in the order of nanometer $\left(1 \mathrm{~nm}=10^{-9}\right.$ $\mathrm{m})$. Since the temperature $T \approx 300 \mathrm{~K}$, we obtain that the characteristic force is $\approx 4 \mathrm{pN}$.

Although the Gaussian chain model can describe the response of a single polymer 
chain under the stretching of a small low force, it is obviously an over-simplified model. One sever simplification is that the distance between two adjacent beads can be extended without bound. The freely jointed chain (FJC) model improves on this point.

\subsection{The freely jointed chain (FJC) model}

In the FJC model, the polymer is composed of a tandem sequence of $N$ rigid rods each of contour length $b$. Each rod can take any orientation irrespective of the orientations of other rods. Under the action of an external force, these rigid rods will align along the force direction, and the total extension $z$ along the force direction reads

$$
z=N b L\left(f b / k_{B} T\right)
$$

where $L(x)$ is the Langevin function defined by

$$
L(x)=1 / \tanh x-1 / x \text {. }
$$

In the limit of $f \ll k_{B} T / b$, Eq. (3) reduces to Eq. (2) of the Gaussian chain model. On the other hand, when $f \gg k_{B} T / b$, Eq. (3) can be re-written in the form

$$
z=N b\left(1-\frac{k_{B} T}{f b}\right) ;
$$

that is, the difference between the total contour length of the polymer and the total extension, $N b-z$, scales with the external force with an inverse linear law (dashed lines of Fig. 1).

The FJC model can be extended to take into account the finite extensibility of each rod. This is achieved via the introduction of a stretching modulus. The extensible FJC model (eFJC) is able to describe the mechanical response of an extended single-stranded DNA (ssDNA) or protein chain fairly good when the external force is of the order of $10 \mathrm{pN}$.

The FJC model also have an important simplification, namely, the bending energy contribution of the polymer chain is not considered. This shortcoming is overcome by the worm-like chain model of the next subsection.

\subsection{The worm-like chain (WLC) model}

The WLC model regards a polymer as an inextensible continuous string of total contour length $L$. The tangential vector of the string at contour length $s$ is denoted as $\hat{\mathbf{t}}(s)$. The coordinate of the string at contour length length $s$ is $\mathbf{r}(s)=\mathbf{r}(0)+$ $\int_{0}^{s} \hat{\mathbf{t}}\left(s^{\prime}\right) \mathrm{d} s^{\prime}$. For each configuration of the string there is an associated bending energy

$$
E[\{\hat{\mathbf{t}}(s)\}]=\int_{0}^{L} \frac{k_{B} T b}{4}\left(\frac{\mathrm{d} \hat{\mathbf{t}}(s)}{\mathrm{d} s}\right)^{2} \mathrm{~d} s .
$$


At the action of an external force of magnitude $f$, the extension $z$ of the string along the force direction can be obtained numerically by transfer matrix method ${ }^{10}$. There also exists an excellent variational expression of $z$ as a function of $f$. This variational expression reads ${ }^{10}$

$$
z=L L(\eta)
$$

where $\eta$ is determined by the external force $f$ by the following equation

$$
\frac{2 f b}{k_{B} T}=\eta+\frac{L(\eta)}{L^{\prime}(\eta)}
$$

with $L^{\prime}(\eta)=\mathrm{d} L(\eta) / \mathrm{d} \eta$. Furthermore, a simple interpolation formula can also be written down for the WLC model ${ }^{11,10}$ :

$$
f=\frac{k_{B} T}{2 b}\left(\frac{1}{(1-z / L)^{2}}-1+4 z / L\right) .
$$

When the external force $f \gg k_{B} T / b$, the force-extension relationship of the WLC model reduces to Eq. (2) of the Gaussian chain model. On the other hand, when $f \gg k_{B} T / b$, it can be shown ${ }^{10}$ that

$$
z=L\left(1-\sqrt{\frac{k_{B} T}{2 f b}}\right) ;
$$

in other words, the difference between the extension $z$ and the total contour length $L$ is proportional to $f^{-1 / 2}$ for large forces (solid lines of Fig. 1). This scaling behavior characterizes the WLC chain model. It was first revealed by Bustamante and coworkers ${ }^{11}$ that, the WLC model is an excellent model for double-stranded DNA at the force regime of $f \leq 10 \mathrm{pN}$. The worm-like chain model with $b \approx 1 \mathrm{~nm}$ can also fit the force-extension curves of extended ssDNA and proteins very well.

What is the physical meaning of the length scale $b$ in Eq. (6)? For the WLC model, usually another quantity, called the bending persistence length $\ell_{b}$, is introduced. For three-dimensional worm-like polymers, the bending persistence length is related to the Kuhn length $b$ by the simple relation that $\ell_{b}=b / 2$. The physical meaning of $\ell_{b}$ is as follows: Suppose that the tangential vector of the string is $\hat{\mathbf{t}}_{0}$ at arc length $s$, then the tangential vector $\hat{\mathbf{t}}_{1}$ at arc length $s^{\prime}$ is correlated to $\hat{\mathbf{t}}_{0}$ through

$$
\left\langle\hat{\mathbf{t}}_{1} \cdot \hat{\mathbf{t}}_{0}\right\rangle=\exp \left(-\frac{\left|s^{\prime}-s\right|}{\ell_{p}}\right) .
$$

The bending persistence length $\ell_{b}$ (or equivalently the Kuhn length $b$ ) is the orientation correlation length of a semiflexible polymer.

\subsection{Comparison of the three polymer models}

As a summary of this section, we demonstrate the force-extension curves predicted by the above-mentioned three polymer elasticity models in Fig. 1 for the case of $b=$ 
$100 \mathrm{~nm}$ and $T=300 \mathrm{~K}$. When the external force $f<0.04 \mathrm{pN}$, all three theoretical curves superimpose onto each other. The differences between these models become significant only when $f \gg 0.04 \mathrm{pN}$.

\section{The over-stretching transition of double-stranded DNA}

It is now widely accepted that, under low or moderate external stretching (force lower than $10 \mathrm{pN}$ ), dsDNA can be regarded as an inextensible semiflexible polymer. At physiological salt conditions, the mechanical response can be well approximated by a worm-like chain of bending persistence $\ell_{b} \simeq 53 \mathrm{~nm}^{1,11,10}$. This is the entropic elasticity regime. In this regime, the external force tends to pull the dsDNA straight, while the thermal noise due to the collision between dsDNA and solution molecules tend to make dsDNA to be in a coiled and curved form. The equilibrium reached between the competition of these two effects determines the total extension of the polymer chain. On the other hand, the dsDNA double helix is not affected by external stretching.

The bending persistence length has two sources of contributions: from the local steric effect between adjacent DNA base-pairs; from the electrostatic repulsive interactions, since dsDNA is a negatively charged polyelectrolyte. Changing the solution salt conditions has an effect on dsDNA bending persistence length.

When the external force is further increased $(f \geq 10 \mathrm{pN})$, dsDNA is more than $95 \%$ aligned along the force direction. In response to the external stretching, therefore dsDNA will be stretched by increasing slightly the vertical distance between two adjacent base-pairs. This leads to a stretching modulus of the order of 1000 $\mathrm{pN}$, a value that is consistent with the bending persistence length of dsDNA ${ }^{12,3}$.

At force $f \approx 70 \mathrm{pN}$, a new phenomenon was observed ${ }^{13,12}$. The total extension of a dsDNA polymer changes from its B-form contour length to about 1.7 times its B-form contour length over a narrow force range of about $5 \mathrm{pN}$. Furthermore, this over-stretching transition is almost reversible, indicating that it is an equilibrium process. The over-stretched DNA is referred to as the S-form DNA in the literature. The nature of the DNA over-stretching transition is extensively investigated by many groups. The most important physical reason for this highly cooperative overstretching transition may be the weak base-pair stacking interaction of dsDNA ${ }^{14}$.

To quantitatively understand the dsDNA over-stretching transition, Zhou and co-authors ${ }^{14,15}$ introduced a semi-microscopic model for dsDNA. This doublestranded polymer model has the following ingredients: (1) the polymer is composed of two inextensible single-stranded chain; (2) these two strands are bound into a double-strand through many lateral base-pairs; (3) between two adjacent base-pairs there is a vertical base-pair stacking interaction. The base-pair stacking potential has the following Lennard-Jones form

$$
U_{\text {stack }}\left(r_{i, i+1}\right)= \begin{cases}\epsilon_{0}\left[\left(\frac{r_{i, i+1}}{r_{0}}\right)^{12}-2\left(\frac{r_{i, i+1}}{r_{0}}\right)^{6}\right] & \text { if right-hand stacking }, \\ \text { constant } & \text { if left-hand stacking }\end{cases}
$$




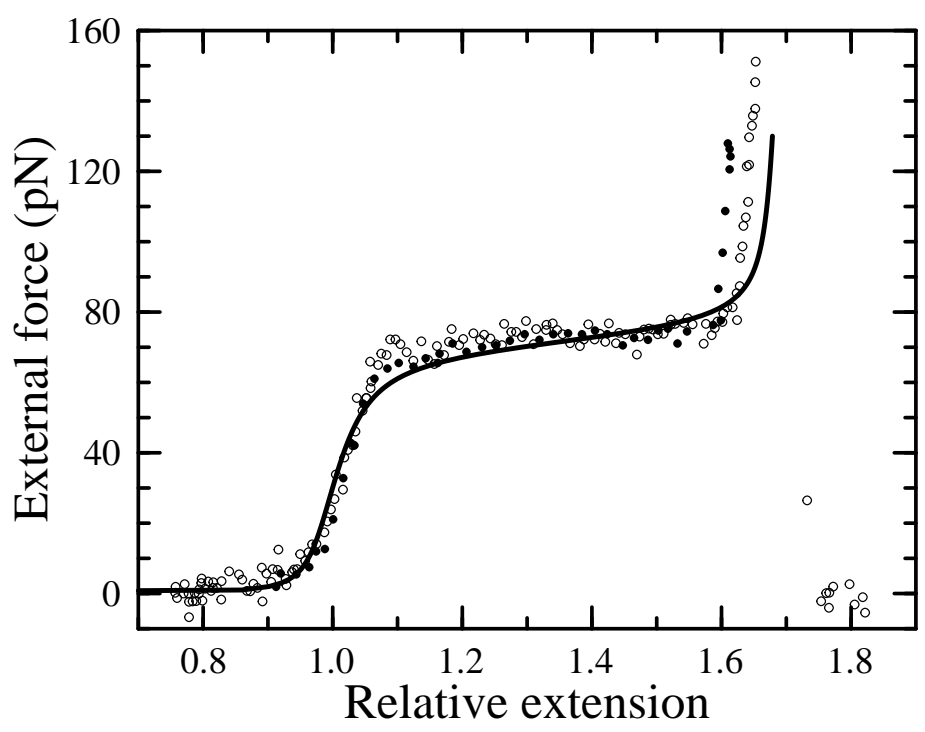

Figure 2. Force-extension response curve for a double-stranded DNA. The open and closed circles are two sets of experimental measurements from Ref. 13; while the solid line is the result of the theoretical model of Ref. 14. The length unit of the horizontal axis is the total contour length of the DNA in its relaxed $B$-form conformation.

In Eq. (12), $\epsilon_{0}$ is the base-pair stacking energy constant; $r_{0}$ is the optimal vertical distance between two base-pairs, $r_{i, i+1}$ is the actual vertical distance between basepairs $i$ and $i+1$. The stacking potential also depends on whether the two adjacent base-pairs are right-handedly stacked or left-handedly stacked. This later consideration ensures that dsDNA in its relaxed state will be a right-handed double-helix.

Zhou and co-authors were able to solve the above-mentioned double-stranded polymer model exactly by using Green function method. The details of their model and their analytical calculations are documented in Ref. 15, here we only mention the main predictions of their theoretical model.

Figure 2 shows the experimental and theoretical force-extension response curves for a long dsDNA. The agreement between the experimental observations of Ref. 13 and the theoretical prediction of the theoretical model is satisfactory, since the theoretical curve of Fig. 2 was obtained with only one fitting parameter. From the viewpoint of base-pair stacking interaction, the over-stretching transition is understood as follows: First, the base-pair stacking interaction is relatively strong, of the order of $10 k_{B} T$ at room temperature. Therefore when the external force is low or moderate, the dsDNA base-pairs are tightly stacked onto each other; this explains why the stretching modulus of dsDNA is very large. On the other hand, the base-pair stacking interaction is a weak interaction, it has only finite interaction range, beyond that range the interaction drops quickly. Therefore, when the inter-base-pair distance increase to a certain critical level, the base-pair stacking 
interaction can no longer withstand the external force; consequently, the doublehelix is untwisted and form a ladder-like structure.

In real situations, the over-stretching transition may be much more complex than was assumed in the above-mentioned model. For example, in the over-stretched Sform, the base-pairs may be tilted with respect to the central axis to gain some residual base-pair stacking. It may also happen that the two strands will be separated from each other by breakage of base-pair hydrogen bonds ${ }^{5}$. In a complete model of dsDNA over-stretching, all these effects should be included.

Since dsDNA is a double-stranded polymer, an important form of deformation is twisting of the double-strand. This twisting causes local torsion in the dsDNA chain. To relax this twisting stress, the conformation of the central line of the dsDNA chain may deform into a curved form, called DNA supercoiling. DNA supercoiling is a very significant biological phenomenon. The model described in this section can also partially explain the behavior of a highly extended and supercoiled dsDNA ${ }^{14,15}$. The numerical work of Zhou and Ou-Yang ${ }^{16}$ also suggested the possibility of a left-handed double-helical DNA structure which is stabilized by external stretching and negative supercoiling.

\section{Denaturation of RNA secondary structures}

RNA is different from dsDNA in that it has only one poly-nucleotide strand. The structure of a RNA chain, on the other hand, can be very complicated, since nucleotide bases along the same chain can form many intra-chain base-pairs. In the absence of external stretching, a RNA chain will fold back at various points to facilitate the formation of base-pairs. The relative positioning of any two base-pairs in a RNA structure can be grouped into three types as shown in Fig. 3: independent, nested, or crossed. If one removes all the crossed base-pairs from a RNA structure in the most economic way (i.e., trying to keep as many base-pairs as possible), the remaining base-pairing pattern is referred to as a secondary structure of the RNA polymer. In most cases, the configurational energy of a RNA structure comes mainly from the configurational energy of its secondary structure. Those crossed base-pairs in a RNA structure further stabilizes the structure; however their energy contribution to the total configurational energy is not comparable to that of the secondary structure. Because of this separation of energy scales, theoretical studies on RNA usually focus on the secondary structures of RNA.

When one end of a RNA polymer is fixed and the other end is pulled with an external force, the structure of the RNA will re-organize so as to make the polymer chain more aligned along the force direction. Experimental observations 17,3,18 revealed that the force-extension response behavior of a RNA polymer is dependent on the RNA sequence. In some experiments ${ }^{3,18}$ a naturally occurring RNA polymer was used for stretching, and the experimental data are consistent with the prediction of a force-induced second-order RNA globule-coil phase transition ${ }^{19}$. This globulecoil transition occurs at force $f \approx 1.0 \mathrm{pN}$, beyond which the relative extension of the 
$\mathbf{a}$

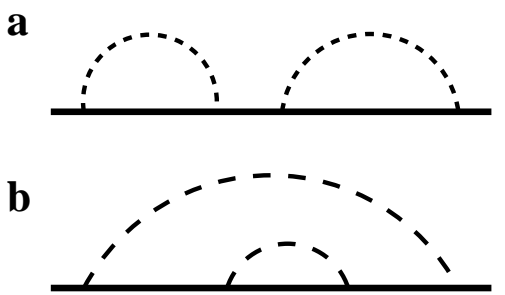

c

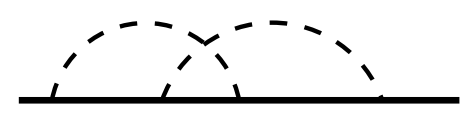

Figure 3. The relative positioning of two base-pairs along a RNA chain: (a) mutual independence; (b) nested; and (c) crossed. Thick line denotes the RNA chain and curved dashed lines denotes the formation of base-pairs.

polymer gradually increases from zero as a function of force ${ }^{3,18}$. On the other hand, Rief and co-authors ${ }^{17}$ performed a RNA stretching experiment using poly-(G-C) or poly- $(\mathrm{A}-\mathrm{T})$ nucleotide chains. They found a force-plateau in the force-extension response curve. Such a force-plateau indicates that the denaturation transition in the RNA polymer is a highly cooperative process.

Zhou and co-workers performed a theoretical study ${ }^{20,21,22}$ on RNA secondarystructure denature, with the aim of understanding the above-mentioned experiments from the same point of view. The major ingredient of their model ${ }^{21}$ is as follows: (1) The RNA is modeled as a linear chain of $N$ beads, with two consecutive beads along the chain being connected by an extensible bond of relaxed length $b$ and certain stretching modulus (the eFJC model of Sec. 2.2). (2) There is short-ranged base-pairing interaction between two beads $i$ and $j$ if the distance between these two beads is smaller than certain value $a_{0}$ (with $a_{0} \ll b$ ) and if both $i$ and $j$ have not yet formed base-pairs with other beads. (3) There is vertical base-pair stacking interaction between two adjacent base-pairs $(i, j)$ and $(i+1, j-1)$. (4) External stretching energy. The statistical mechanical property of this self-interacting polymer was explored by analytical calculations ${ }^{21}$ and also by Monte Carlo simulations 22. Here we describe the main conclusions of their work (see Fig. 4).

When the mean base-pair stacking potential is negligible, the RNA globulecoil transition is determined mainly by the average strength of the base-pairing interaction. The transition is a continuous phase-transition in the thermodynamic limit $^{19}$. On the other hand, when the mean base-pair stacking potential is increased, the denaturation process of RNA becomes more and more co-operative (see Fig. 4), and a force-plateau will appear in the force-extension response curve. The work of Zhou and co-authors ${ }^{21}$ demonstrated that the nearest-neighbor base-pair stacking potential has a dramatic effect in controlling the cooperativity of the denaturation of RNA.

The mean base-pair stacking potential can be controlled by sequence. If the 


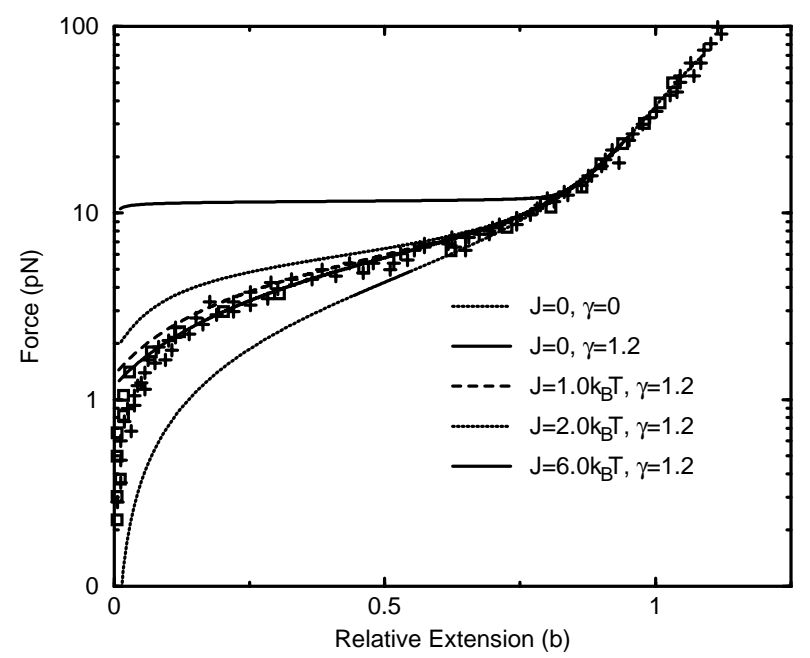

Figure 4. Force-induced RNA secondary-structure denature. The parameter $\gamma$ is related to basepairing interaction, while the parameter $J$ is the mean base-pair stacking strength ${ }^{21}$. Symbols are experimental observations of Refs. 3, 18, while lines are theoretical calculations.

RNA sequence is relatively random, the probability to form two consecutive basepairs $(i, j)$ and $(i+1, j-1)$ is relatively low, and therefore the mean base-pair stacking potential is low; on the other hand, if the RNA chain is highly designed, then base-pair stacking possibility will be also very high.

\section{The $\beta$-sheet secondary structure of proteins}

The protein folding problem is one of the major issues in biophysics. At the level of secondary structures, there are $\alpha$-helices and $\beta$-sheets. It has been known for more than forty years that the $\alpha$-helix-coil transition is not a real phase transition in the thermodynamic limit ${ }^{23}$. However, the nature of the $\beta$-sheet-coil transition is not yet completely settled. Working on a simplified flexible lattice-polymer, References 24, 25, 26 all predicted that the $\beta$-sheet-coil transition is a second-order phase transition in the thermodynamic limit. In this section we investigate a similar $2 \mathrm{D}$ partially directed lattice model of protein $\beta$-sheet denaturation. We find that the order of the $\beta$-sheet-coil transition is controlled by the bending stiffness $\Delta$ of the model polymer: it is second-order for a polymer with exactly zero bending stiffness $(\Delta \equiv 0)$, while it changes to be first-order when the bending stiffness $\Delta$ is non-zero.

\subsection{The model and the major analytical procedure}

We study a 2D partially directed lattice polymer model as shown in Fig. 5. A chain of $N$ identical units is located on a square lattice. The length of the bond connecting two consecutive monomers $i$ and $i+1$ is fixed to $a_{0}$, while the direction of bond in $-\mathbf{z}_{0}$ is prohibited. If any two monomers $i$ and $i+m(m \geq 3)$ occupy nearest 


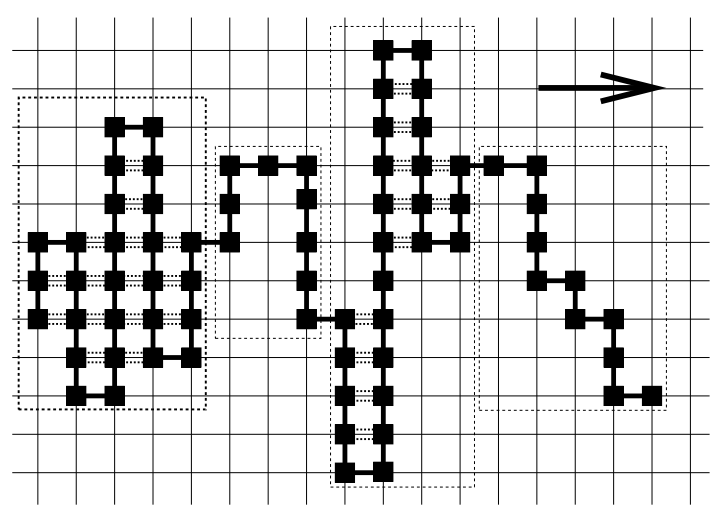

Figure 5. An 2D lattice polymer model with contacting interaction, bending stiffness, and external stretching. The arrow shows the $\mathbf{z}_{0}$ direction.

neighboring lattice sites, an attractive energy of magnitude $\epsilon$ is gained. Besides this 'hydrogen-bonding' interaction, we also consider the bending energy of the polymer chain. An energy penalty of magnitude $\Delta$ is added to each local direction change of the chain ${ }^{27}$.

We follow Lifson's method ${ }^{28}$ to calculate the free energy density of the polymer. For this purpose, a given configuration of the $2 \mathrm{D}$ chain is divided into a linear sequence of $\beta$-sheet segments and coil segments. A $\beta$-sheet segment is defined as a folded segment of $n_{\beta} \geq 2$ consecutive columns, in which contacting interactions exist between any two adjacent ones. Two consecutive $\beta$-sheet segments are separated by a coil segment, which is a segment of $n_{\mathrm{c}} \geq 0$ consecutive columns in which all monomers are free of contacts. For example, the configuration shown in Fig. 5 has two $\beta$-sheets and two coils.

Under the action of an external force $f$, the energy of a $\beta$-sheet of $n_{\beta}$ columns is

$$
E_{\beta}=-\epsilon \sum_{j=1}^{n_{\beta}-1} \mathrm{v}\left(l_{j}, l_{j+1}\right)-n_{\beta} f a_{0}+2\left(n_{\beta}-1\right) \Delta,
$$

where $l_{j}$ is the number of monomers in the $j$-th column of the $\beta$-sheet, and $\mathrm{v}\left(l_{j}, l_{j+1}\right)=\min \left(l_{j}, l_{j+1}\right)-1$. On the other hand, the configurational energy of a coil segment is

$$
E_{c}=-n_{\mathrm{c}} f a_{0}+m_{\mathrm{c}} \Delta,
$$

where $n_{\mathrm{c}}$ and $m_{\mathrm{c}}$ are, respectively, the total number of columns and the total number of bends in the configuration.

To calculate the total partition function of the model system, we proceed by first calculating the partition functions of $\beta$-sheets and coils separately, and then combining them to obtain the free energy density of the whole polymer. The calculation details will be reported elsewhere ${ }^{29}$, here we focus on the final results. 


\subsection{Analytical and simulational results}

Qualitatively speaking, the $\beta$-sheet-coil transition is caused by the competition between monomer-monomer attraction and configurational entropy: Formation of contacts lowers the energy, but it requires monomers to be aligned and close to each other, thereby decreasing the polymer's degrees of configurational freedom. When the monomer-monomer attraction is much stronger than thermal energy, the polymer takes $\beta$-sheeted conformations to maximize the number of contacts. At the other limit of high temperatures, the polymer is in completely disordered coil states with maximal entropy. The analytical work of Zhou and co-authors ${ }^{29}$ have confirmed this picture. It has been predicted that the $\beta$-sheet-coil structural transition is a real phase transition process in the thermodynamic limit. The order of this phase transition depends on whether the bending stiffness of the polymer is flexible (with $\Delta=0$ ) or semi-flexible (with $\Delta>0$ ).

In the case of $\Delta=0$, the $\beta$-sheet-coil transition is second-order. The relative extension versus temperature curve at zero temperature is shown in Fig. 6a, and the relative extension versus force curve at constant temperature $T=0.591 \epsilon$ is shown in Fig. 6b. When there is no external force, a second-order globule-coil phase transition occurs at $T_{\mathrm{gc}}(0)=0.8205 \epsilon$. The force-induced globule-coil transition at constant temperature is also second-order. These results are in accordance with Refs. 30, 26.

In the case of positive bending stiffness, the $\beta$-sheet-coil transition becomes a first-order phase transition process. For example at $\Delta=0.5 \epsilon$ and $f=0$, the relative extension jumps from zero to 0.193 at the transition temperature $T_{\mathrm{gc}}=1.209 \epsilon$ (Fig. 6a). Such a large jump is also observed in the force-induced transition (Fig. 6b). A non-zero bending stiffness therefore is able to dramatically enhance the cooperativity of the globule-coil phase transition. This may be partially understood in the following way. A positive bending energy significantly decreases the configurational entropy of a coil segment. Consequently the globule-coil transition will occur at higher temperature and higher force, and once the polymer is unfolded it favors those highly elongated configurations which have fewer bends.

It is interesting to notice that a non-zero bending stiffness of the polymer changes the nature of the collapse transition from being second-order to being first-order. This conclusion is in agreement with an earlier exact enumeration study ${ }^{27}$, and it is also consistent with the mean-field calculations of Orland and co-workers ${ }^{31}$. It is well known that, in the 2D Ising model the paramagnet-ferromagnet phase transition changes from being second-order to being first-order under the action of a non-zero external magnetic field. What surprises us is that, in the polymer system such a qualitative change is caused not by an external field (such as the external force), but by an internal (microscopic) parameter, the bending stiffness $\Delta$. At this point, additional MC simulation work is needed for the study of more realistic models, e.g., a self-attractive and semiflexible self-avoiding $2 \mathrm{D}$ or $3 \mathrm{D}$ chain. It is also highly desirable to perform real $2 \mathrm{D}$ polymer collapse experiments. For 

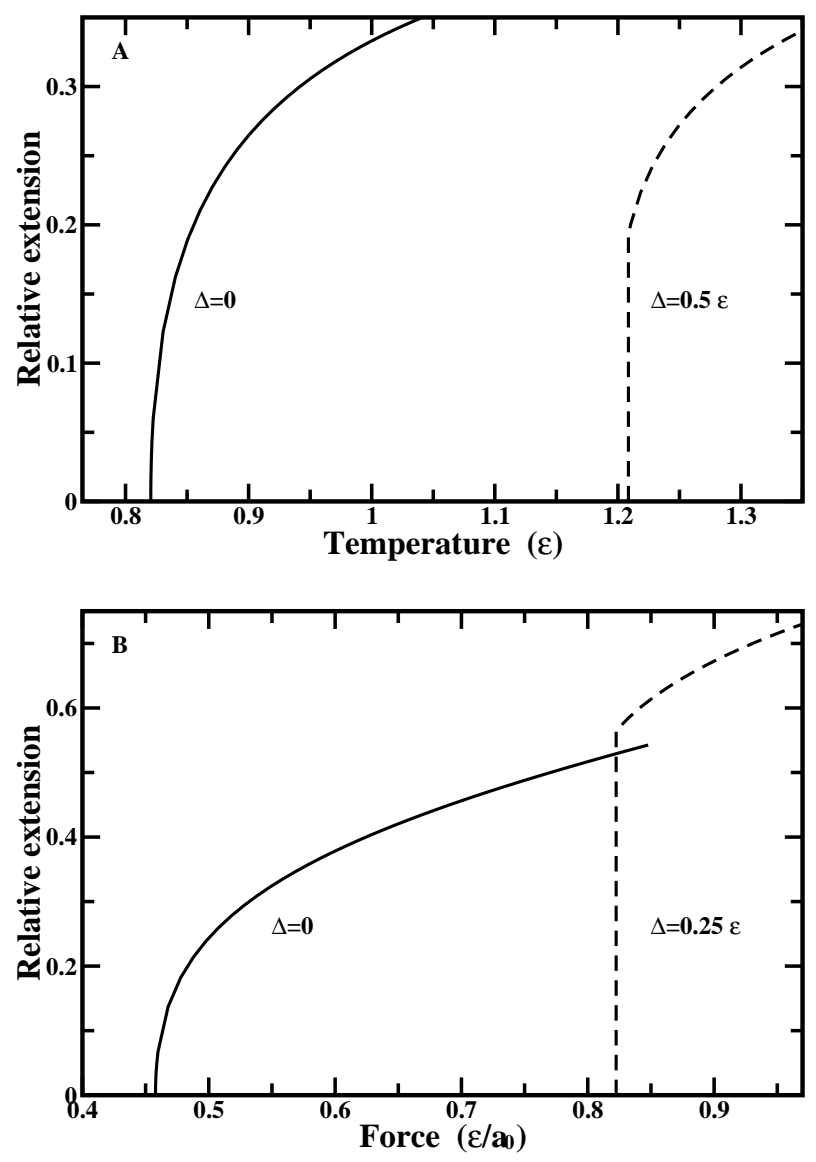

Figure 6. $\quad \beta$-sheet unfolding. (A) temperature-extension curve at force $f=0$; (B) Force-extension curve at temperature $T=0.590928 \epsilon$. Solid lines correspond to a flexible polymer $(\Delta=0)$, dashed lines correspond to a semiflexible polymer $(\Delta>0)$.

example, a long polypeptide chain, say poly(Glycine), can be attached to a mobile lipid bilayer ${ }^{32}$ and its configurations can be recorded in real-time and manipulated by controlling temperature or external force.

\section{Elasticity of spider capture silk}

The capture silk is a natural material produced by orb-web weaving spiders. Spiders rely on it to entrap flying preys ${ }^{33}$. Like the spider dragline silk and many other naturally occurring silks, the capture silk has a high tensile strength that is comparable to steel; but unlike steel, it is also extremely elastic, with the ability to be stretched to almost ten times its relaxed contour length without breaking ${ }^{34,35}$. This perfect combination of strength and extensibility conveys a high degree of toughness to the capture silk: its breakage energy per unit weight is more than twenty times 
that of a high-tensile steel ${ }^{34}$. On the other hand, the mechanism behind spider silk's remarkable strength and elasticity is still largely missing, partly because of the difficulty to obtain high-quality crystallized structures of silk proteins.

In a recent experiment, Hansma and co-workers ${ }^{35}$ attached capture silk mesostructures (probably composed of a single protein molecule) or intact capture silk fibers to an atomic force microscopy tip and recorded the response of the samples to external stretching force. They found a remarkable exponential relationship between the extension $x$ and the external force $f$,

$$
f \propto \exp (x / \ell)
$$

where the length constant $\ell$ is a fitting parameter. In the spider capture silk experiment, the exponential behavior was observed at both fluid and air within a force range from about $10^{0}$ piconewton $(\mathrm{pN})$ to about $10^{6} \mathrm{pN}^{35}$. The exponential force-extension curve is significantly different from the predictions of simple polymer models as reviewed in Sec. 2.

Equation (15) indicates the following: (i) Because the capture silk is highly extensible, a great amount of extra length must have been stored in its relaxed form. (ii) Since extension increases with force logarithmically, some fraction of the stored length must be easy to be pulled out, some fraction be harder to be pulled out, and till some other fraction be even harder to be pulled out. To model this kind of heuristic cascading responses, a hierarchical chain model was suggested for spider capture silk in Ref. 36 (see Fig. 7). In the hierarchical chain model, the polymer is composed of many basic structural motifs; these motifs are then organized into a hierarchy, forming structural modules on more and more longer length scales. At the deepest hierarchy level $h_{m}$, the structural motifs could be $\beta$-sheets, $\beta$-spirals, helices or microcrystal structures. The interactions among some of these motifs are much more stronger than their interactions with other motifs, therefore they form a structural module at the hierarchy level $\left(h_{m}-1\right)$. These level- $\left(h_{m}-1\right)$ modules are then merged into level- $\left(h_{m}-2\right)$ modules through their mutual interactions. This merging process is continued; and finally at the global scale, the whole spider silk string is regarded as a single module of the hierarchy level $h=0$.

When the spider capture silk is under an external stretching, the total extension of a structural module at the hierarchy level $h=0$ can be decomposed into two parts. First, the weak bonds between those level- $(h=1)$ sub-modules of this module may break; the relative positions of these sub-modules will then be displaced, leading to an elongation of the level- 0 module. This contribution of extension will eventually saturate when all the weak bonds between these level-1 sub-modules are completely destroyed. However, there is another source of elongation, namely that each of these level-1 sub-module will have an internal deformation under the stretching. The deformation of a level-1 sub-module, in turn, can be further decomposed into two parts; .... A semi-quantitative calculation was performed in Ref. 36 based on this picture of elongation cascade. The results are shown in Fig. 7. This figure suggests that the exponentially observed exponential force-extension behavior of 


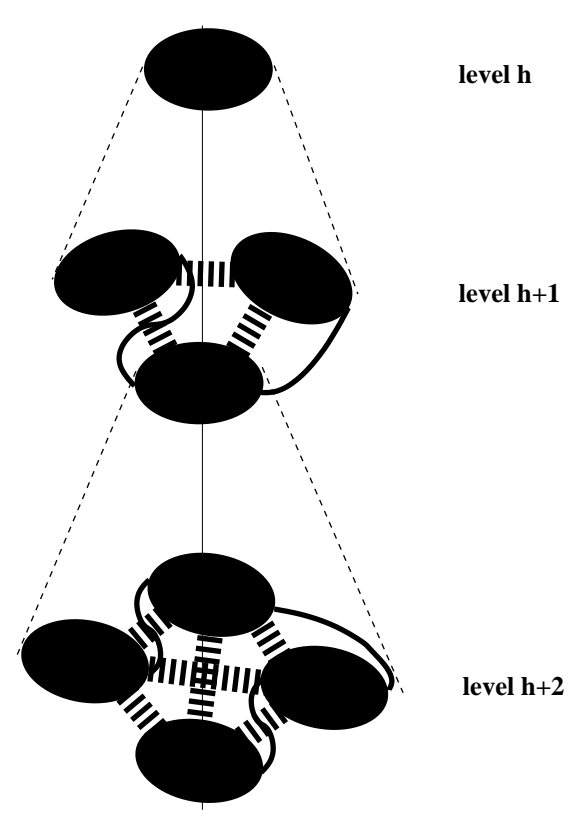

Figure 7. The hierarchical chain model for spider capture silk. At each hierarchy level $h$ a structural module is composed of a tandem sequence of $m_{h}$ submodules $M_{h+1}$ of hierarchy level $h+1$. The thick broken lines between submodules of each hierarchy level indicate the existence of sacrificial bonds.

spider capture silk can be explained by the hierarchical chain model. According to this model, the response of the spider capture silk to external perturbations is in a hierarchical manner. If the external force is small, only those structural units of length scale comparable to the whole polymer length will be displaced and rearranged; structural units at short and moderate length scales will remain intact. As the external perturbation is increased, additional structural units at more and more shorter length scales are also deformed. Through such a hierarchical organization, a single polymer chain can respond to a great variety of external conditions; at the same time, it is able to keep its degree of structural integrity as high as possible. This hierarchical modular structure also indicates a broad spectrum of relaxation times. The modules at the shorter length scales will have much shorter relaxation times and will be refolded first when the external force decreases. This gap in relaxation times ensures that, after extension, the spider capture silk will return to its relaxed state gradually and slowly. This is a desirable feature for spider capture silk, because a too rapid contract following the insect's impact would propel the victim away from the web.

The simple hierarchical chain model, while appealing, needs further experimental validation. This model seems to be supported by recent genetic sequencing efforts. By analyzing the cDNA sequence of the major protein of spider capture silk, the flagelliform protein, it was revealed that the amino-acid sequence of flagelliform has 


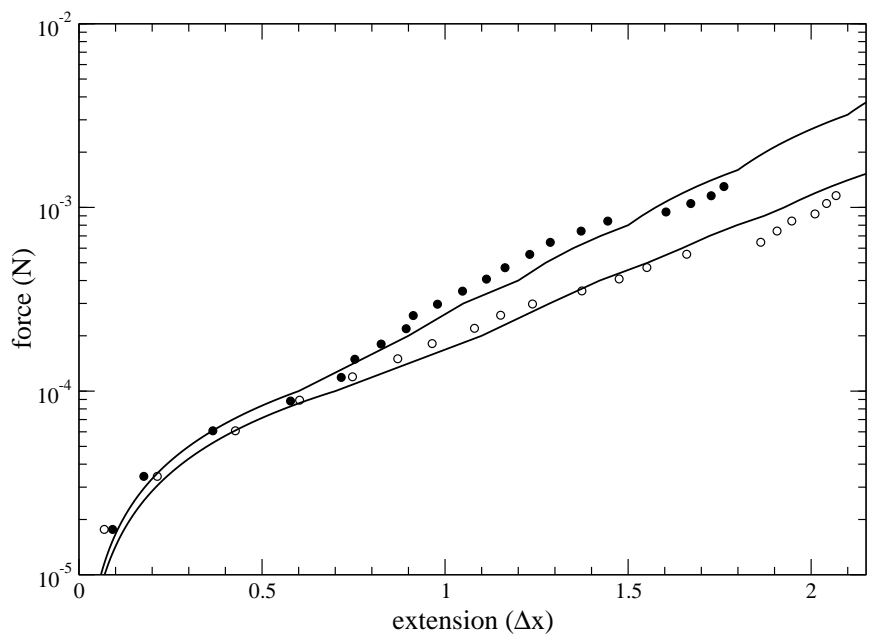

Figure 8. Exponential force-extension relationship for the hierarchical chain model. Lines are theoretical calculations ${ }^{36}$ with two different sets of parameters and symbols are experimental data of Ref. 35 .

a hierarchy of modularity $37,38,39$. At the sequence level, the structures of spider capture silks therefore have the potential to be hierarchically organized. More experimental as well as large-scale numerical simulation work are needed to fully understand the structural organization of spider capture silks.

\section{Conclusion}

In this review paper, we briefly described some recent theoretical work on the mechanical properties and structural transitions of biopolymers. We have discussed DNA over-stretching transition, RNA secondary-structure denature, protein $\beta$-sheet unfolding, and also structural organization principle of spider capture silk. From these studies, we get the impression that weak non-covalent bonds and interactions, convey both stability and flexibility to a biopolymer system.

\section{Acknowledgments}

I am grateful to Sanjay Kumar, Reinhard Lipowsky, Zhong-Can Ou-Yang, Yang Zhang and Jie Zhou for support and collaboration. This review paper is based on an talk given by the author at the Second International Symposium on the Frontier of Applied Mathematics, in honor of Prof. C. C. Lin at his 90th birthday. 


\section{References}

1. S. B. Smith, L. Finzi and C. Bustamante, Science 258, 1122 (1992).

2. H. Zhou, Y. Zhang and Z.-C. Ou-Yang, Theoretical and computational treatments of dna and rna molecules, in Handbook of Theoretical and Computational Nanotechnology, eds. M. Rieth and W. Schommers (American Scientific Publishers, California, 2005) pp. 1-69.

3. C. Bustamante, S. B. Smith, J. Liphardt and D. Smith, Curr. Opin. Struct. Biol. 10, $279(2000)$.

4. T. R. Strick, G. Charvin, N. H. Dekker, J.-F. Allemand, D. Bensimon and V. Croquette, C. R. Physique 3, 595 (2002).

5. M. C. Williams and I. Rouzina, Curr. Opin. Struct. Biol. 12, 330 (2002).

6. C. Bustamante, Z. Bryant and S. B. Smith, Nature 421, 423 (2003).

7. J.-F. Allemand, D. Bensimon and V. Croquette, Curr. Opin. Struct. Biol. 13, 266 (2003).

8. F. Bueche, Physical Properties of Polymers (Interscience, New York, 1962).

9. P. J. Flory, Statistical Mechanics of Chain Molecules (Interscience, New York, 1969).

10. J. F. Marko and E. D. Siggia, Macromolecules 28, 8759 (1995).

11. C. Bustamante, J. F. Marko, E. D. Siggia and S. Smith, Science 265, 1599 (1994).

12. S. B. Smith, Y. Cui and C. Bustamante, Science 271, 795 (1996).

13. P. Cluzel, A. Lebrun, C. Heller, R. Lavery, J.-L. Viovy, D. Chatenay and F. Caron, Science 271, 792 (1996).

14. H. Zhou, Y. Zhang and Z.-C. Ou-Yang, Phys. Rev. Lett. 82, 4560 (1999).

15. H. Zhou, Y. Zhang and Z.-C. Ou-Yang, Phys. Rev. E 62, 1045 (2000).

16. H. Zhou and Z.-C. Ou-Yang, Modern Phys. Lett. B 13, 999 (1999).

17. M. Rief, H. Clausen-Schaumann and H. E. Gaub, Nature Struct. Biol. 6, 346 (1999).

18. B. Maier, D. Bensimon and V. Croquette, Proc. Natl. Acad. Sci. USA 97, 12002 (2000).

19. A. Montanari and M. Mézard, Phys. Rev. Lett. 86, 2178 (2001).

20. H. Zhou, Y. Zhang and Z.-C. Ou-Yang, Phys. Rev. Lett. 86, 356 (2001).

21. H. Zhou and Y. Zhang, J. Chem. Phys. 114, 8694 (2001).

22. Y. Zhang, H. Zhou and Z.-C. Ou-Yang, Biophys. J. 81, 1133 (2001).

23. D. Poland and H. A. Scheraga, Theory of Helix-Coil Transitions in Biopolymers: Statistical Mechanical Theory of Order-Disorder Transitions in Biological Macromolecules (Academic Press, New York, 1970).

24. R. Brak, A. J. Guttmann and S. G. Whittington, J. Phys. A: Math. Gen. 25, 2437 (1992).

25. A. L. Owczarek and T. Prellberg, Physica A 205, 203 (1994).

26. A. Rosa, D. Marenduzzo, A. Maritan and F. Seno, Phys. Rev. E 67, p. 041802 (2003).

27. S. Kumar and D. Giri, Phys. Rev. E 72, p. 052901 (2005).

28. S. Lifson, J. Chem. Phys. 40, 3705 (1964).

29. H. Zhou, J. Zhou, Z.-C. Ou-Yang and S. Kumar, Collapse transition of twodimensional flexible and semiflexible polymers, Unpublished, (2006).

30. P. Grassberger and H.-P. Hsu, Phys. Rev. E 65, p. 031807 (2002).

31. S. Doniach, T. Garel and H. Orland, J. Chem. Phys. 105, 1601 (1996).

32. B. Maier and J. O. Rädler, Phys. Rev. Lett. 82, 1911 (1999).

33. F. Vollrath, Sci. Am. 266, 70 (1992).

34. J. M. Gosline, P. A. Guerette, C. S. Ortlepp and K. N. Savage, J. Exp. Biol. 202, 3295 (1999).

35. N. Becker, E. Oroudjev, S. Mutz, J. P. Cleveland, P. K. Hansma, C. Y. Hayashi, D. E. Makarov and H. G. Hansma, Nature Materials 2, 278 (2003). 
36. H. Zhou and Y. Zhang, Phys. Rev. Lett. 94, p. 028104 (2005).

37. P. A. Guertte, D. G. Ginzinger, B. H. F. Weber and J. M. Gosline, Science 272, 112 (1996).

38. C. Y. Hayashi and R. V. Lewis, J. Mol. Biol. 275, 773 (1998).

39. C. Y. Hayashi and R. V. Lewis, Science 287, 1477 (2000). 